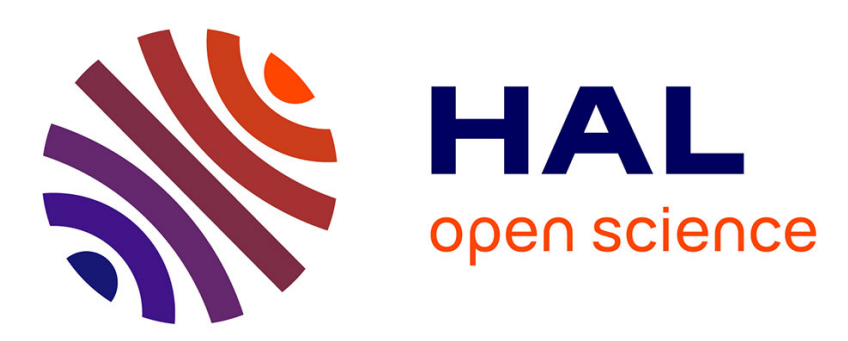

\title{
Angular difference measure between tomographic projections taken at unknown directions in $2 \mathrm{D}$
}

Minh-Son Phan, Etienne Baudrier, Loïc Mazo, Mohamed Tajine

\section{To cite this version:}

Minh-Son Phan, Etienne Baudrier, Loïc Mazo, Mohamed Tajine. Angular difference measure between tomographic projections taken at unknown directions in 2D. 2014 IEEE International Conference on Image Processing (ICIP), Oct 2014, Paris, France. 10.1109/ICIP.2014.7025348 . hal-01910194

\author{
HAL Id: hal-01910194 \\ https://hal.science/hal-01910194
}

Submitted on 31 Oct 2018

HAL is a multi-disciplinary open access archive for the deposit and dissemination of scientific research documents, whether they are published or not. The documents may come from teaching and research institutions in France or abroad, or from public or private research centers.
L'archive ouverte pluridisciplinaire HAL, est destinée au dépôt et à la diffusion de documents scientifiques de niveau recherche, publiés ou non, émanant des établissements d'enseignement et de recherche français ou étrangers, des laboratoires publics ou privés. 


\title{
ANGULAR DIFFERENCE MEASURE BETWEEN TOMOGRAPHIC PROJECTIONS TAKEN AT UNKNOWN DIRECTIONS IN 2D
}

\author{
Minh-Son Phan, Étienne Baudrier, Loïc Mazo, Mohamed Tajine \\ ICube, University of Strasbourg, CNRS \\ 300 Bd Sébastien Brant - CS 10413 - 67412 ILLKIRCH, FRANCE
}

\begin{abstract}
This paper introduces a new measure for estimating the angular difference between two tomographic projections belonging to a set of projections taken at unknown directions. The measure is potential for many applications such as projection refinement or projection classification, which are important in the process of tomographic reconstruction. Our measure relies on the construction of a neighborhood graph for projection moments, the calculus of the angular difference for neighboring projections and the computation of geodesics on this graph. The accuracy and the robustness of our measure is shown on a test database including 50 2D gray-level images at different resolutions and with different levels of noise.
\end{abstract}

Index Terms - tomography, unknown direction, Euclidean distance, angular difference measure

\section{INTRODUCTION}

The problem of tomographic reconstruction for unknown direction projections has been widely examined in $[1,2,3,4,5]$. There are generally two steps: first estimating the projection directions, then reconstructing the object from the estimated directions. This process depends on the measurement of distance between two projection vectors in which the Euclidean distance is often used. For example, in [1], Coifman et al. compute the Euclidean distance between the acquired projections for the purpose of their classification and their direction estimation. In [2], Van Heel computes the Euclidean distance between the acquired projections and those generated from the reconstruction model. The objective is to refine the reconstruction model. Another measure used to compare the projections is the cross-correlation coefficient as shown in [3], but there is no difference between the Euclidean distance and the cross-correlation coefficient in practice (see [6] for more details).

On the other hand, we observe that the Euclidean distance is not invariant under the rotation of the object even for the two close projections. More precisely, we can obtain many different values of the Euclidean distance between projections, taken at the two fixed directions when rotating the object around its centroid. Therefore, there can be errors when using the Euclidean distance in some situations such as the projection refinement or the projection classification.

To overcome this problem, we propose in this paper a new measure that estimates the projection angular difference by using the properties of projection moments. The organization of the paper is as follows. In Sec. 2, we provide the background notions that are used in Sec. 3 for solving the problem of angular difference estimation. Sec. 4 deals with the problem of noise. The performance of our measure is shown for 2D images in Sec. 5. Finally, conclusion and perspective are presented in Sec. 6.

\section{BACKGROUND NOTIONS}

We first present the definition of the projection as follows.

Definition 1 (Projection). Let $f: \mathbb{R}^{2} \rightarrow[0,1]$ a measurable function with compact support. Let $\theta \in \mathbb{R}$. The projection $\mathcal{P}_{f}(\theta)$ in the direction $\theta$ is defined by

$$
\mathcal{P}_{f}(\theta)(x)=\int_{\mathbb{R}} f\left(\mathrm{R}_{-\theta}(x, y)\right) \mathrm{d} y
$$

where $\mathrm{R}_{\theta}$ is the rotation by the angle $\theta$ around the origin.

Note that $\mathcal{P}_{f}(\theta)(x)=\mathcal{P}_{f}(\theta+\pi)(-x)$. Thus we can assume that $\theta \in\left[-\frac{\pi}{2}, \frac{\pi}{2}\right)$.

The definition of the moment of a projection is:

Definition 2 (Moment of projection). Let $d \in \mathbb{N}$. The $d$ th order moment of a projection $\mathcal{P}_{f}(\theta)$ is given by

$$
\mu_{f, d}(\theta)=\int_{\mathbb{R}} x^{d}\left(\mathcal{P}_{f}(\theta)(x)\right) \mathrm{d} x .
$$

Moments of projection are trigonometric polynomials.

Property 1 ([4]). Let $d \in \mathbb{N}$. The $d$ th order moment of a projection $\mathcal{P}_{f}(\theta)$ can be presented as

$$
\mu_{f, d}(\theta)=\sum_{\substack{0 \leq k \leq d \\ k \equiv \bar{d} \bmod 2}} a_{k} \cos (k \theta)+b_{k} \sin (k \theta)
$$

where $a_{k}, b_{k} \in \mathbb{R}$.

In the following, for simplicity, we write $\mathcal{P}$ and $\mu_{d}$ instead of $\mathcal{P}_{f}$ and $\mu_{f, d}$. 


\section{ESTIMATION OF THE ANGULAR DIFFERENCE BETWEEN TWO PROJECTIONS}

\subsection{Problem of angular difference estimation}

Let $f$ be a function whose centroid is at the origin, $n \in \mathbb{N}$, $\Theta=\left\{\theta_{1}, \ldots, \theta_{n}\right\} \subseteq\left[-\frac{\pi}{2}, \frac{\pi}{2}\right)$ be a set of unknown directions and $\Pi=\left\{\mathcal{P}\left(\theta_{i}\right) \mid 1 \leq i \leq n\right\}$ be the set of their associated projections. The angular difference between two projections is:

$$
\mathrm{D}_{\text {ang }}\left(\mathcal{P}\left(\theta_{i}\right), \mathcal{P}\left(\theta_{j}\right)\right)=\left|\theta_{i}-\theta_{j}\right|
$$

Our main goal in this work is to estimate the angular difference between any two projections of the set $\Pi$.

In [7], Salzman shows that it is possible to compute the direction $\theta$ associated with the projection $\mathcal{P}(\theta)$ by using the following equation:

$$
\sin ^{2}(\theta)=\frac{\mu_{2}^{\max }-\mu_{2}(\theta)}{\mu_{2}^{\max }-\mu_{2}^{\min }}
$$

where $\mu_{2}^{\max }=\max _{\theta \in \mathbb{R}} \mu_{2}(\theta)$ and $\mu_{2}^{\min }=\min _{\theta \in \mathbb{R}} \mu_{2}(\theta)$. Nevertheless, there are two possible values of $\theta$ in (2) since the function sin is odd. To address this problem, Salzman uses the odd order moment which can disambiguate the angle value. However, due to the high slope of the function arcsin near the abscissa 1 , the calculus of $\mathrm{D}_{\text {ang }}\left(\mathcal{P}\left(\theta_{i}\right), \mathcal{P}\left(\theta_{j}\right)\right)$ derived from (2) would not be robust to noise when the moment of one of the projections is close from $\mu_{2}^{\min }$. Instead, we give a criterion for finding the closest projections from a given one (Sec. 3.2). Then, we linearize the angular difference for close projections (Sec. 3.3). Finally, we measure the angular difference between any two projections as the length of the shortest path between these projections in the graph whose vertices are the projections $\mathcal{P}\left(\theta_{i}\right)$ and whose edges connect close angle projections, weighted by the measure found in Sec. 3.3.

\subsection{Searching for the neighbors of projection}

The aim of this section is a method for selecting (in the projection set $\Pi$ ) the neighbors of a given projection. Since the moments are non-monotonic, close moments are not synonymous of close angles. Hence, for each moment $\mu_{d}(\theta)$, we need to find an interval $\mu_{d}(\theta) \pm \varepsilon_{d}(\theta)$ in which can be found, with some chosen probability $p$, at least one moment corresponding to a close projection. Assuming that the $\theta$ are independent, uniformly distributed, random variables in $\left[-\frac{\pi}{2}, \frac{\pi}{2}\right)$, we derive from (2), that

$$
\varepsilon_{2}(\theta) \approx 2 \delta \sqrt{\left(\mu_{2}^{\max }-\mu_{2}(\theta)\right)\left(\mu_{2}(\theta)-\mu_{2}^{\min }\right)},
$$

where

$$
\delta=\frac{\pi}{2}\left(1-(1-p)^{\frac{1}{n-1}}\right)
$$

For the moment $\mu_{1}$, we obtain from (1)

$$
\varepsilon_{1}(\theta) \approx \delta \sqrt{\left(\left(\mu_{1}^{\max }\right)^{2}-\left(\mu_{1}(\theta)\right)^{2}\right)},
$$

and, for $d \geq 3$, using Bernstein's inequality for Fourier series,

$$
\varepsilon_{d}(\theta) \leq d \delta \max \left\{\left|\mu_{d}(\theta)\right|\right\}
$$

In conclusion, (3), (4) and (5) allow us to find the neighbors of each projection by cross-checking the information given by moments of distinct orders.

The problem of measuring the angular difference between two neighboring projections is presented in the following section.

\subsection{Estimation formulae}

Eq. (2) is rewritten as

$$
\mu_{2}(\theta)=\mu_{2}^{\max } \cos ^{2}(\theta)+\mu_{2}^{\min } \sin ^{2}(\theta)
$$

We develop the finite Taylor series for $\mu_{2}$ at $\theta$

$$
\mu_{2}(\theta+\Delta \theta)=\mu_{2}(\theta)+\frac{\mathrm{d} \mu_{2}}{\mathrm{~d} \theta} \Delta \theta+o(\Delta \theta)
$$

where $\frac{\mathrm{d} \mu_{2}}{\mathrm{~d} \theta}=2\left(\mu_{2}^{\max }-\mu_{2}^{\min }\right) \sin (\theta) \cos (\theta)$. In addition, we have from (2)

$$
\sin (\theta) \cos (\theta)= \pm \frac{\sqrt{\left(\mu_{2}^{\max }-\mu_{2}(\theta)\right)\left(\mu_{2}(\theta)-\mu_{2}^{\min }\right)}}{\mu_{2}^{\max }-\mu_{2}^{\min }} .
$$

We then obtain from (6) and (7)

$$
\Delta \theta= \pm \frac{\mu_{2}(\theta+\Delta \theta)-\mu_{2}(\theta)}{2 \sqrt{\left(\mu_{2}^{\max }-\mu_{2}(\theta)\right)\left(\mu_{2}(\theta)-\mu_{2}^{\min }\right)}}+o(\Delta \theta) .
$$

Thus the approximation of $|\Delta \theta|$ for small $\Delta \theta$ is given by

$$
|\Delta \theta| \approx \frac{\left|\mu_{2}(\theta+\Delta \theta)-\mu_{2}(\theta)\right|}{2 \sqrt{\left(\mu_{2}^{\max }-\mu_{2}(\theta)\right)\left(\mu_{2}(\theta)-\mu_{2}^{\min }\right)}} .
$$

However, when $\mu_{2}(\theta)$ approaches $\mu_{2}^{\max }$ or $\mu_{2}^{\min }$, the denominator of (8) approaches 0 . This will increase the approximation error in (8). Therefore, two other approximation formulae are developed for these cases.

For small $\Delta \theta$, one has $\Delta \theta \approx \sin (\theta+\Delta \theta)-\sin (\theta)$. Then, from (2), we derive that, if $\theta$ and $\theta+\Delta \theta$ have the same sign,

$$
|\Delta \theta| \approx \frac{\left|\sqrt{\mu_{2}^{\max }-\mu_{2}(\theta+\Delta \theta)}-\sqrt{\mu_{2}^{\max }-\mu_{2}(\theta)}\right|}{\sqrt{\mu_{2}^{\max }-\mu_{2}^{\min }}}
$$

and if $\theta$ and $\theta+\Delta \theta$ have opposite signs,

$$
|\Delta \theta| \approx \frac{\left|\sqrt{\mu_{2}^{\max }-\mu_{2}(\theta+\Delta \theta)}+\sqrt{\mu_{2}^{\max }-\mu_{2}(\theta)}\right|}{\sqrt{\mu_{2}^{\max }-\mu_{2}^{\min }}} .
$$


When $\theta$ and $\theta+\Delta \theta$ have opposite signs, there is at least one projection $\left(\mu_{2}^{\max }\right)$ whose angle $(\theta=0)$ is closer from $\theta$ than $\theta+\Delta \theta$. So, since, at the first stage, we are interested in finding pairs of projections whose angles are as close as possible (see Sec. 3.2), we are allowed to set (9) as the default formula when $\mu_{2}(\theta)$ approaches $\mu_{2}^{\max }$.

Alike, when $\mu_{2}(\theta)$ approaches $\mu_{2}^{\min }$, we obtain the following formula

$$
|\Delta \theta| \approx \frac{\left|\sqrt{\mu_{2}(\theta+\Delta \theta)-\mu_{2}^{\min }}-\sqrt{\mu_{2}(\theta)-\mu_{2}^{\min }}\right|}{\sqrt{\mu_{2}^{\max }-\mu_{2}^{\min }}} .
$$

In conclusion, the angular difference between any two close projections can be estimated by the so-called Angular Difference Measure through (8), (9) and (10):

$$
\operatorname{ADM}\left(\mathcal{P}\left(\theta_{i}\right), \mathcal{P}\left(\theta_{j}\right)\right)= \begin{cases}(10), & \text { if } \mu_{2}\left(\theta_{i}\right) \approx \mu_{2}^{\min } \\ (9), & \text { if } \mu_{2}\left(\theta_{i}\right) \approx \mu_{2}^{\max } \\ (8), & \text { otherwise }\end{cases}
$$

Note that the ADM depends on the whole projection set for $\mu_{2}^{\min }$ and $\mu_{2}^{\max }$ determination.

Remark 1. In Section 3, $\mu_{d}^{\max }$ and $\mu_{d}^{\min }$ appear in Eq. (3), (4), (5), (8), (9), (10). Nevertheless, the exact value of $\mu_{d}^{\max }$ and $\mu_{d}^{\text {min }}$ are not known since they are estimated from the (finite) set of projections $\Pi$. Even if it is not possible to give the proof here due to the lack of place, it is possible to show that the hypothesis "the angular difference $\Delta \theta$ between close angles is very small in probability" leads to an error on $\mu_{d}^{\max }$ and $\mu_{d}^{\min }$ less than $(\Delta \theta)^{2}$. Then the error on $\mu_{d}^{\max }$ and $\mu_{d}^{\min }$ do not modify the formulae obtained above.

Eventually, the angular difference between two projections which are not close to each other is computed via Dijkstra's shortest path algorithm [8]. Our method is summarized in Algorithm 1 in which the orders of moments of projections are 1,2, 3 and 5 since they are sufficient to find the neighbors of each projection.

\section{DEALING WITH NOISE}

In reality, the projections are often contaminated by noise that is modeled as a white centered Gaussian noise. We first present here the noise robustness of our method without preprocessing, then we present an efficient denoising method that is used as preprocessing step. Our method noise robustness comes from the use of moments of projection. It is known that the sum of Gaussian independent variables is also a Gaussian variable. One can show that the $d$ th order moment of the noisy projection $x \longmapsto \mathcal{P}(\theta)(x)+\mathcal{N}_{x}$, where $\mathcal{N}_{x}$ is a Gaussian noise with zero mean and a variance $\sigma^{2}, \forall x \in[-1,1]$, follows the Gaussian distribution with a mean $\mu_{d}(\theta)$ and a variance $\int_{-1}^{1} x^{2 d} \sigma^{2} d x=\frac{\sigma^{2}}{d+1 / 2} \leq \sigma^{2}$. So, using the moments of projection for angular difference estimation can help

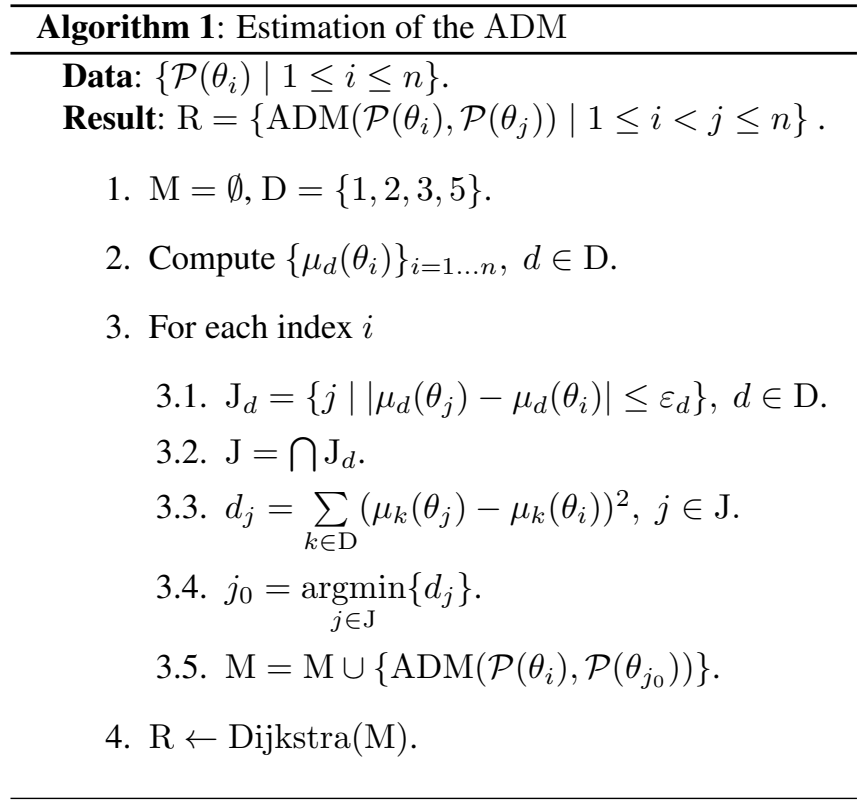

us to reduce the noise in a ratio of $d+1 / 2$. However, in case of significant noise, this reduction is not sufficient. Therefore, we employ a projection denoising method developped by Singer and Wu in [9] as a denoising step. However, only the first step of their method which is based on the PCA decomposition [10], is employed to denoise the set of tomographic projections. Even if it is not the whole step, it is called here the Singer-Wu method. The efficiency of the denoising step is shown in Fig. 5.

\section{EXPERIMENTS}

Our method is applied for a set of 50 images with different sizes $(32 \times 32,64 \times 64,128 \times 128$ and $256 \times 256$ pixels) as those shown in Fig. 1. In the first experiment, given $\mathcal{I}=\left(0^{\circ}, 2^{\circ}\right]$, we randomly generated 300 pairs $(\theta, \Delta \theta) \in$ $\left[-90^{\circ}, 90^{\circ}\right) \times \mathcal{I}$. The associated projections $\mathcal{P}(\theta)$ and $\mathcal{P}(\theta+$ $\Delta \theta)$ are then computed. The ADM and the Euclidean distance between $\mathcal{P}(\theta)$ and $\mathcal{P}(\theta+\Delta \theta)$ are estimated. Our objective is to measure the dispersion of the ADM (resp. the Euclidean distance) with respect to $\Delta \theta$. In order to measure the dispersion, we use the variance-to-mean ratio (VMR):

$$
\mathrm{VMR}=\frac{1}{k} \sum_{i=0}^{k-1} \frac{\sigma_{i}^{2}}{\mu_{i}}
$$

where $\sigma_{i}^{2}, \mu_{i}$ are the variance and the mean of the ADM (resp. the Euclidean distance) for $\Delta \theta \in(\alpha i, \alpha(i+1)]$, where $\alpha=$ 0.1 is the sample step such that $\mathcal{I}=\bigcup_{i=0}^{k-1}(\alpha i, \alpha(i+1)]$ and consider that $k=\left\lfloor\frac{\sup (\mathcal{I})-\inf (\mathcal{I})}{\alpha}\right\rfloor$. The VMR must be 


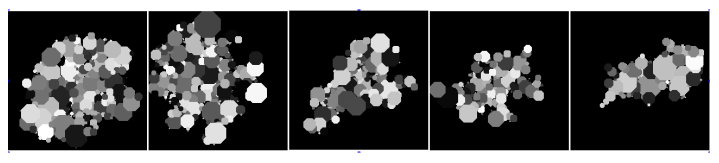

Fig. 1: Image data

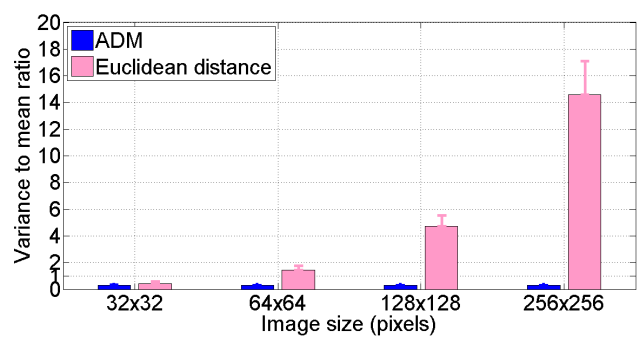

Fig. 2: First experiment : mean and standard deviation of the VMR for the Euclidean distance and the ADM, according to image sizes.

lower than 1 for a negligible dispersion. The result is illustrated in Fig. 2.

Clearly, the VMRs of the Euclidean distance are higher than 1 from the image size $64 \times 64$ pixels, whereas the VMRs of the ADM are lower than 1 for all image sizes. An example of the Euclidean distance and the ADM computed from an image of size $256 \times 256$ pixels is also illustrated in Fig. 3. We see that the dispersion of the Euclidean distance ( $x$ axis) with respect to $\Delta \theta$ ( $y$ axis) in Fig. 3a is greater than the ADM in Fig. 3b.

In the second experiment, given an image and 300 random projections with $\theta \in\left[-90^{\circ}, 90^{\circ}\right)$, the ADMs between all pairs of projections are computed, using Algorithm 1. The VMR is evaluated with a sample step $\alpha=0.5$. We also compare the ADM with the Euclidean distance and the angular difference estimated by (2), noted as the Salzman's measure. As seen in Fig. 4, the VMRs of the ADM are very small compared to the ones of the Euclidean distance and the Salzman's measure. Then, a Gaussian noise with a given SNR is added to the projections. The results of estimating the ADM before and after using the Singer-Wu method described in Sec. 4 are shown in Fig. 5 for images of size $256 \times 256$ pixels. The VMRs after using this denoising step are lower than before and the means of the VMRs are still lower than 1 at $\mathrm{SNR}>18 \mathrm{~dB}$.

\section{CONCLUSION}

We present in this paper a new measure for estimating the angular difference between two unknown direction projections. We first present the selection of neighboring projections from their moments. Then formulae of the moment based angular difference for neighboring projections are given. These measures are used for constructing the projection graph whose

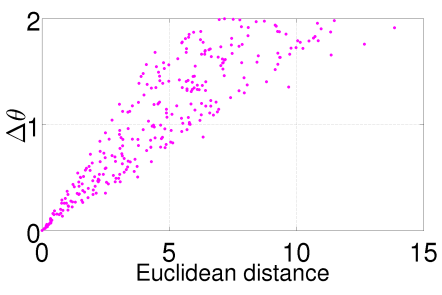

(a)

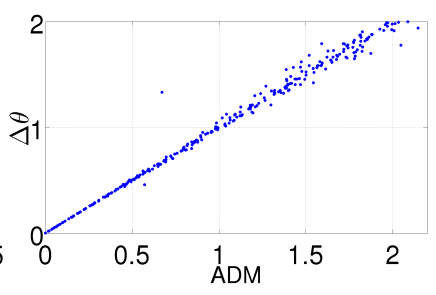

(b)
Fig. 3: First experiment : dispersion of the Euclidean distance and the ADM, from an image of size $256 \times 256$ pixels.

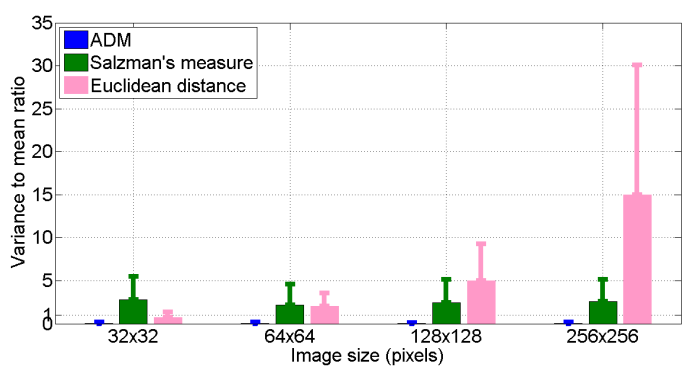

Fig. 4: Second experiment : mean and standard deviation of the VMR for the ADM, the Euclidean distance and the Salzman's measure, according to image sizes.

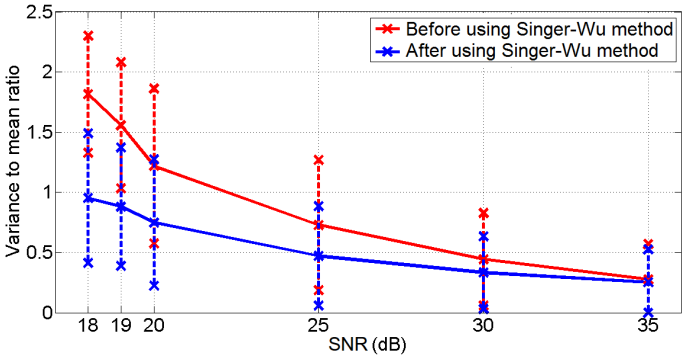

Fig. 5: Second experiment : mean and standard deviation of the VMR for the ADM, from images of size $256 \times 256$ pixels, according to the SNR before and after using the Singer-Wu method.

edges connect the neighboring projections, weighted by our measure. The angular differences between any two projections in the graph are then computed by the Dijkstra's shortest path algorithm. Our measure has been evaluated on a set of 2D images and the results shown that our measure performes better than the Euclidean distance and the Salzman's measure at the different tested image resolutions. Moreover they show that our measure is robust at moderate levels of noise. Our measure is promising for many applications such as projection refinement, projection classification, etc. Our future work is to extend the measure for 3D and improve its robustness at the higher levels of noise. 


\section{REFERENCES}

[1] R. R. Coifman, Y. Shkolnisky, F. J. Sigworth, and A. Singer, "Graph laplacian tomography from unknown random projections," IEEE Trans Image Process, vol. 17, no. 10, pp. 1891-1899, Oct. 2008.

[2] M. Van Heel, "Multivariate statistical classification of noisy images (randomly oriented biological macromolecules)," Ultramicroscopy, vol. 13, no. 1-2, pp. 165183, 1984.

[3] — , "Angular reconstitution: a posteriori assignment of projection directions for 3D reconstruction," Ultramicroscopy, vol. 21, no. 2, pp. 111-123, 1987.

[4] S. Basu and Y. Bresler, "Feasibility of tomography with unknown view angles." IEEE Trans Image Process, vol. 9, no. 6, pp. 1107-22, Jan. 2000.

[5] C. Fillion, A. Daurat, B. Naegel, G. Frey, and E. Baudrier, "A new ab initio reconstruction method from unknown-direction projections of $2 \mathrm{~d}$ binary set," in Proc. of Int Conf on Image Process, IEEE, Ed., 2013.

[6] J. Frank, Three-dimensional electron microscopy of macromolecular assemblies: visualization of biological molecules in their native state. New York: Oxford Univ. Press, 2006.

[7] D. B. Salzman, "A method of general moments for orienting 2D projections of unknown 3D objects," Comput Vision Graph, vol. 50, no. 2, pp. 129-156, May 1990.

[8] E. W. Dijkstra, "A note on two problems in connexion with graphs," Numerische Mathematik, vol. 1, no. 1, pp. 269-271, Dec. 1959.

[9] A. Singer and H. T. Wu, "Two-dimensional tomography from noisy projections taken at unknown random directions," J Imaging Sci, vol. 6, no. 1, pp. 136-175, Feb. 2013.

[10] I. T. Jolliffe, Principal component analysis. New York: Springer, 2002. 